\title{
Effective Integration of Mobile Applications in the Context of Education System
}

\author{
Sathya Priya Balasegaran \\ Department of Computer \\ Science \& Engineering \\ Sri Manakula Vinayagar \\ Engineering College \\ Madagadipet, Puducherry, India
}

\author{
Kalaranjani Jayakumar \\ Department of Computer \\ Science \& Engineering \\ Sri Manakula Vinayagar \\ Engineering College \\ Madagadipet, Puducherry, India
}

\begin{abstract}
With the advancement of wireless communication and portable devices, mobile users not only access information ubiquitously, but also use mobile devices for business transactions. Users exchange location, preferences and purchase details for discounts and privileges, in commercial applications. Mobile applications for educational purposes have plausible prospects. Applications for an educational gaming purpose, a classroom management control, a reference tool and an online study group can be developed. Massive Open Online Courses (MOOCs) aim to bring education to the entire world online, with access to university level courses, thereby promising a quality educational experience. This paper presents the concept applied in M-Commerce transactions, wherein a real time mobile application for an educational portal is developed, to enable e-learning in a wireless environment. It utilizes an Android platform for learners to access and refer online study materials, test themselves through mobile devices, and view their evaluation results. Consequently, intra and interdepartmental group analyses are generated, for assessment by academic heads. It also provides an opportunity to improve students' performance, for enlightening themselves intellectually and for sharing the knowledge gained among peers. This proposed model could be implemented as a MOOC across groups of institutions, interactive study forums and networking communities.
\end{abstract}

\section{General Terms}

Mobile Computing, Wireless Networks, Online Course Content, Mobile Application, Result Analysis, Performance Analysis.

\section{Keywords}

Mobile Educational Portal, Portable Educational Applications, Mobile Learning Initiative, Cellular Courses Portal, Mobile Assisted Learning, Mobile Course Management System, Mobile Virtual Learning, Mobile-aided Performance Analysis.

\section{BACKGROUND}

\subsection{Mobile Computing}

Mobile computing [1] is a fast growing technology that stimulates normal computer operations and interactions with the help of portable systems. It is a type of information management platform wherein unrestricted data is available "on the go". Mobile computing constitutes mobile hardware, mobile software, and mobile communication. Mobile hardware includes portable devices such as handsets and digitals assistants. Mobile software is embedded in mobile applications and programs, enhancing the functionality of the hardware. The communication module essentially covers all aspects of the entities needed in a wireless environment, such as a wireless network, the necessary communication infrastructure such as Global System for Mobile Communication (GSM), including data formats and standard protocols.

\subsubsection{Advantages}

\subsubsection{Saves Time}

Information available on request helps answer customer centric queries, complete tasks within deadlines, and exchange information from any location.

\subsubsection{Supports Cloud Computing}

Critical documents can be stored on an online server, which could be accessed by travelling business professionals from time to time.

\subsubsection{Monitors Data}

Tracking devices used in freight and baggage control maintain records of positions and activities of moving objects and their attributes.

\subsection{Mobile Data}

Mobile Data is simply data transmitted via air waves between communicating devices, which are usually mobile terminals such as smartphones and tracking devices. Mobile data transmission applies in satellite communication, RFID (Radio Frequency Identification) technologies, email, paging, instant messaging and mobile web data access amongst others.

Wireless applications have been used in improving worker productivity, providing care to differently abled children, providing dynamic data in transportation and public safety, automation of field activities, pricing and customer problem alerts, indexing user preferences, insurance, healthcare, ERP (Enterprise Resource Planning) systems and Business Intelligence (BI). Mobile data communications help key staff in an organization operate remotely and keep tabs on crucial data in an efficient manner.

\subsection{Mobile Applications}

A mobile application [2] is software written to be deployed on a compatible mobile device, combining the best features of 
similar services being provided through desktop computers. Mobile apps (applications) are pre-installed on smart devices during manufacture, delivered as web applications or procured through distribution platforms such as the Windows Phone Store, Google Play and BlackBerry App World, to name a few. These apps are being used for general productivity and information retrieval such as email, calendar, contacts, stock market, weather information and news alerts.

In the past, such applications may not have been that prominent because of the limited hardware resources of the devices. However, these days, owing to customers' demands and preferences, they are readily customizable for the sake of the users.

\subsection{Mobile Application Development}

Mobile application development is the window where one embeds coding procedures, add-ins and plug-ins, interactive controls and texts into task specific applications, to run on personal and enterprise digital assistants, tablets and mobile phones. Mobile application development is similar to web application development, the only difference being the former has to be ensured of its compatibility with the target device. For instance, one could develop gaming apps for an iPhone, for a unique gaming experience with its accelerometer.

\subsection{Wireless Communications}

Wireless communications fall under the category in which electromagnetic signals replace physical wires to carry data using the required connectivity protocols. The sender and the receiver devices, which broadcast these signals, set up a communication path between them through which bytes of data are propagated rapidly, without a tangible connection.

Examples of wireless communication control include EDGE (Enhanced Data GSM Environment), GPRS (General Packet Radio Service) and WAP (Wireless Application Protocol). This feature is also harnessed in recent technologies such as Infrared and Bluetooth, and in Wireless Network Communication, of which Wi-Fi technology is a part.

\subsection{Wi-Fi Technology}

A Wi-Fi network comprises a group of connected devices that exchange data wirelessly, up to a distance of 100 feet apart. In technical terms, it depicts the features of a Wireless Local Area Network (WLAN), complying to pre-established IEEE (Institute of Electrical and Electronics Engineers) 802.11 standards.

An access point plays the role of a network hub or an interface, it broadcasts signals that are captured and decoded by computer terminals in the wireless network. Such a close knit network, usually containing a single access point to a larger network, supports free mobility of nodes within the coverage area. A Wi-Fi environment, also known as a 'Hotspot' can be set up in public spaces and business networks, to permit Internet access to countless number of terminals.

\subsection{Mobile Analytics}

Mobile analytics is a fairly new concept, which claims to offer solutions in business intelligence and it assists as a tool to monitor business operations. Some of its functions include events tracking, monitoring mobile behaviours, observing visitors attributes and their device specifications, sending notifications to prospective customers based on their actions, mobile marketing, and improvising mobile based transactions to be at par with traditional web transactions.
Statistics have shown that the scope of Business Intelligence coupled with Mobile Analytics markets is expected to be between $\$ 600$ and $\$ 2.1$ billion in 2015. One major difference between mobile analytics and standard web analytics is that the former yields much more precise information on locationbased data, allowing service providers and site owners to trace user activities easily.

\section{STRUCTURE OF THE PAPER}

The paper is organized as follows:

Section 3 discusses the Related Work done in the mobile computing domain. Section 4 presents the Existing System and its issues. Section 5 introduces the Proposed System, followed by the System Architecture in Section 6. The Implementation details, describing the modules in the system have been thoroughly explained in Section 7 and Section 8 elaborates on the management of the complete system. Section 9 illustrates the results and further analysis done in the proposed concept. Section 10 concludes the paper, followed by a suggestion for future enhancement in Section 11.

\section{RELATED WORK}

A mobile telecom network monitors the movement of mobile users and its mobility management has been used to study human movement issues [3]. Standard outputs such as handover rates, call arrival rates, call holding time and call traffic measured in a mobile telecom network are used to study human movement patterns, thereby aiding to handle issues such as vehicular traffic and spreading of diseases. Methods have been devised to minimise the cost of dynamic location of mobile nodes, by observing a node's whereabouts in two dimensional spaces in wireless networks [4]. We can generate association rules among items in a database, for instance, in the set of items in a customer's purchase transaction. Analysis of past transactions improves the quality of business decisions to maximize profits. Such databases can be enhanced by the concept of antecedent and consequent items. For instance, if a customer buys bread and butter (antecedents), there are 90\% chances that he buys milk (consequent). Thus the consequent items would improve in sales and profits. Rules can have one item in the consequent and a union of items in the antecedent. In fact, algorithms have been developed to integrate movement and purchase patterns in a potential customer [5], using sequential patterns generated by his/her mobile device.

Applications in mobile commerce also associate and predict user movement and purchase transactions [6], based on the similarities among stores and items a customer visits and purchases. This data can be used to promote, advertise and make recommendations about stores to prospective customers. Mobile patterns can be generated from huge mobile logs. However, such transactions lack temporal and behavioural pattern details in a differential mobile user, hence prediction about mobile patterns may not be feasible. Algorithms have been proposed to form user clusters based on mobile transactions and make suitable predictions about subsequent patterns, by considering user behaviour and temporal properties simultaneously [7]. It further proposes to segment time intervals where similar user and mobile characteristics exist. The suitable time intervals are generated, based on similarities in mobile behaviour.

Bayesian networks have been successfully applied in forecasting and information retrieval and an algorithm has been proposed to construct these networks from functional 
dependencies (FD) and relational databases [8]. Such methods which utilize an effective mining process are helpful, especially when essential data is inadequate. The business models in Internet e-commerce have revolutionized business processes by converting traditionally available resources into electronic versions. Some of them include E-shop, E-auctions, third party marketplace, value chain integration model, portal model, information brokerage, virtual organizations, etc. A formal model as a case study shows the typical workflow in an electronic bank transfer and its properties in transactional consistencies have been identified.

Bayesian networks are also being used in cooperative tracking of nodes [9] for determining their positions in a MANET (Mobile Ad hoc Network). This effective tracking in large coverage areas using minimal number of nodes could be helpful in critical applications such as disaster relief operations, expedition of historic artefacts, remote monitoring and defence mechanisms amongst others. An experimental analysis using a small number of anchor nodes equipped with external GPS receivers for an indoor tracking operation has been conducted and its efficiency observed.

Data mining methods that collect information from online social networks as well as mobile sensors, in order to study real-life human dynamics have been developed [10]. These methods observe user input data such as the routine behaviour of an individual, in addition to structures that perform in regular time intervals and under specific time constraints, and generate behaviour patterns. The temporal patterns thus generated can be used for monitoring social networks and routing efficiency, to provide deeper insights into social relationships. In fact, a cell phone could be used more than just as a communication device, to manage human social networks and mine suitable data from them [11]. It proposes a "SmartPhonebook", which caches users' social connections to manage relationships. If a candidate user is in a certain situation, it alerts a close acquaintance or friend of the user, based on the degree of closeness existing between each other. The system also analyses the user's emotional states and behaviours, and uses Bayesian networks to handle ambiguity in the mobile social network. The efficiency of the proposed system has been experimented with MS Windows Mobile 2003 SE Platform on Samsung SPH-M4650 smartphone, and tested with real-world input values.

Mobile sensors installed in GPS-equipped taxis have revolutionised the system of driving [12]. These sensors guide a taxi driver in choosing the appropriate routes, on keen observation of the traffic rhythm, and they adapt themselves to the driver's own style of driving. This concept explores a new data mining capability that involves mining path traversal patterns in a distributed environment containing documents and objects linked together. It has its application in on-line services, where users travel from one object to other using hyperlinks to seek information. GPS-equipped mobile phones also create personal profiles of users [13] based on the current location, places of interest, close contacts and other aspects through continuous tracking. GPS systems are common in outdoor environments and have been adopted in the latest smartphones and tablet computers. However, due to the complexity of infrastructure requirements, no distinguished solutions have been developed for indoor environments. One of the conventional mechanisms, the Received Signal Strength (RSS) coupled with Wi-Fi technology [14] can be used for radio fingerprinting. A set of RSS from a nearby access point, is collected and stored with corresponding location information in a radio map. The mobile user then measures RSS at his/her current position, which is then compared with the fingerprint map. This helps to find the best-matching RSS, and the details are reported back to the user. The proposed system has been tested in an Android smartphone, with accurate location results.

Mobile Search engines capture users' preferences in the form of concepts by mining their click through data [15]. It has also prototyped such a model in a Google Android platform, which has been worked upon in our project development. Adaptability is the key determining factor to all kinds of vendor environment, and it determines how technologies help integrate the enterprise infrastructure. Such an engine offers quick effective business solutions, thus increasing productivity. Besides, it ensures data confidentiality and security from unauthorized access or loss at all times owing to its reliable security features.

Mobile technologies have been developed to serve differently abled children through appropriate health advices from potential care providers [16]. A practical model has been presented, that allows parents of such children to discuss with volunteers in a social network, and gain timely assistance regarding health issues. The burden of building mobile commerce systems is not laid upon a single entity; rather it can be built on the functionalities rendered by others. We can design a framework mainly focusing on the user plane that governs the overall interactions among the mobile applications layer, the middleware layer and the network layer in the infrastructure. Express Q, WAP, and the W3C standards are types of mobile middleware that unite apps, tools, networks and technologies as a common interface for users.

Mobile service systems have the capability towards effectively mining a special request based on dynamic user behaviours [17]. Four necessary attributes: the mobile user, the movement location, the timestamp, and the service request are taken into account. The system makes suitable predictions on a user by studying the behaviour and access patterns. In a ubiquitous environment, intelligent mobile agents capture the same by communicating with the users. The user behaviour supports effective mining methods such as the temporal mobile access pattern. This pattern discovers temporal behaviour patterns associated with location and request services, using mobile agent systems.

Multicast applications that route messages based on the locations of sending and receiving nodes are quite infeasible, owing to the difficulties in implementing them in a MANET in a real time scenario. Algorithms have been proposed, considering possible strategies to overcome the standard limitations in a MANET [18]. They propose a method to propagate messages within a defined geographical range around the sender, allowing the receivers to perform the message matching. The next strategy suggests using directed acyclic graphs (DAGs) to direct messages to the appropriate receivers, this time authorizing the senders to perform message matching. A third strategy combines the best qualities of the above two proposals. It specifies a particular range for transmitting the messages and queries, with intermediate repeater nodes fixed at suitable points in the network, meant for matching and routing. Through experimental evaluation, it has been inferred that the third hybrid strategy is favourable to perform such multicast message transactions depending upon the locations of the communicating nodes. Data collection latency may be higher in wireless networks, due to slow speed of data exchange 
among mobile elements. Schemes have been presented to reduce the path length between nodes in a network [19], thus lowering the data collection latency considerably. This ensures fast transfer of data in wireless networks.

\section{EXISTING SYSTEM}

The following key areas use present generation mobile devices.

\subsection{Medical Applications}

Human anatomical studies, involving dissections of a cadaver up to 40 layers "virtually", 3-D visualizations of images obtained from CT and MRI scans, are now conducted on a mobile device such as the iPhone. With the actual storage of the item's data set being stored on a remote or cloud server, users can now zoom, orient, manipulate and view high resolution biomedical images on their devices. This interactive technology also enables doctors to discuss with patients health issues and problems associated with their body conditions.

\subsection{Portable Libraries}

Library services are being introduced as mobile applications for students in universities. Students can search for catalogues, journals and computers availability with the help of a suitable online reference librarian, to assist them in their queries.

\subsection{Cellular Campus}

In universities, freshmen and seniors exchange information about course content, course offerings and campus experiences among themselves, in a mobile environment. Video lectures are shared via live podcasting by academicians and students update each other on current affairs using video blogs and mobile versions of major news networks. Besides, researchers find it easy to handle smart devices to analyse, compare and present experimental observations and academic research findings.

\subsection{Issues and Challenges}

Mobile applications have been limited to commercial and business environments, health care, mobile web systems, service management etc. However, it has not yet been fully explored in the education sector. M-Commerce is an emerging trend that has shown positive results. By overcoming common limitations, mobile learning has the potential to expand even further, reach out to the masses and enlighten motivated young minds to innovate and change the world.

\section{PROPOSED SYSTEM}

Today's students are comfortable in a digital world. Students prefer to study at their own paces and learning capacities, sometimes even at odd moments such as while travelling, sitting in a park or playground, or even at a waiting lounge. Our paper describes a user-friendly mobile interface that enables students to take up course related tests from their own portable devices in real time. Wi-Fi technology is employed to connect various devices, installed with the mobile application. Using this we introduce the concept of online test taking via mobile devices as this can bring in a turning point in the education system.

Our system does the following operations:

- Uploading test questions for individual test takers by a centralized server.
- Providing a test platform where students can solve questions related to the subjects they learn.

- Section wise and chapter wise analysis of an individual test taker's performance.

- Providing online course materials for students, to view and study in their mobile devices.

- Providing an opportunity for students to improve their performance via improvement tests, should they need any (i.e., if they score below the cutoff).

- Analyzing the group results via graphical simulations department wise and among students, useful for staff members and test in-charges.

\section{SYSTEM ARCHITECTURE}

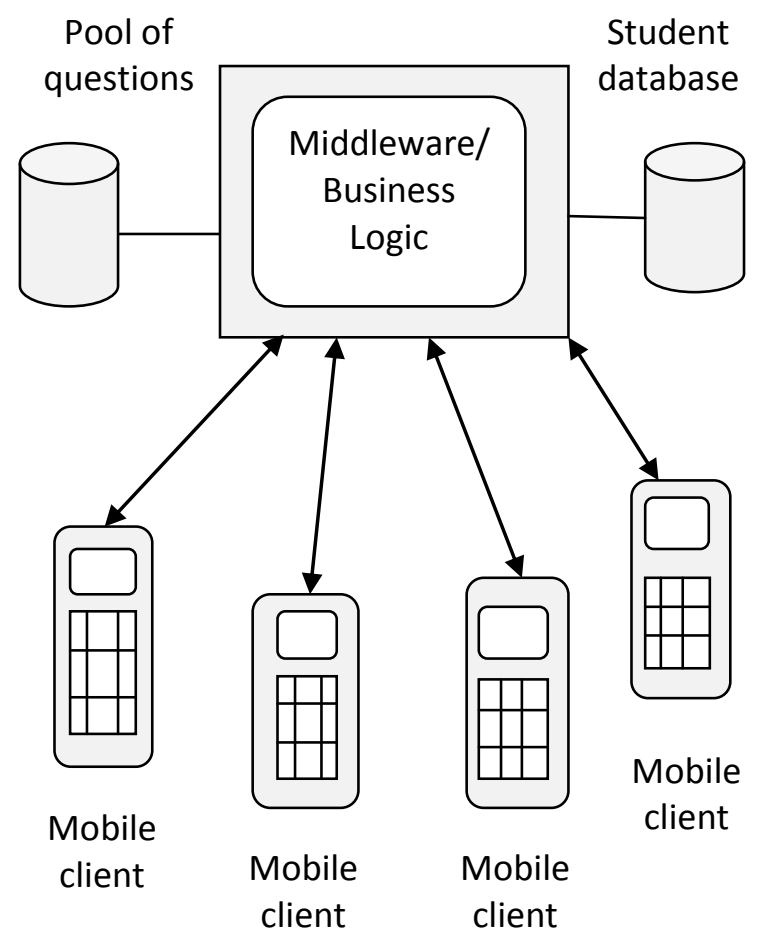

Figure 1: Architecture Diagram

\subsection{Architecture Description}

The architecture includes four types of components:

\subsubsection{Pool of questions}

It is nothing but the question database in which questions are grouped section and chapter wise, and further into two categories: core tests and improvement tests questions.

\subsubsection{Mobile Clients}

The common platform is installed on an individual device, providing the basic User Interface (UI) and the environment for the test portal.

\subsubsection{Student Database}

It stores the registration, performance and test records of individual students. 


\subsubsection{Business Logic/Middleware}

The middleware is the web server which maintains the interoperability with the devices and runs the application. The business logic directs students to take the necessary steps to improve their performance (i.e., course content, improvement questions), if found unsatisfactory.

\section{IMPLEMENTATION DETAILS}

Following is the list of modules in our system:

- Registration Section

- $\quad$ Online Test Round

- Evaluation Report Generation

- Group Analysis

\subsection{Registration Section}

- The test takers are required to register their personal details such as name, year, contact number, email id etc., at their respective online portals. The portals store the data with the server for future use.

- Registration is done through the mobile device. The student indicates his/her department which plays a key role in delivering the appropriate technical content.

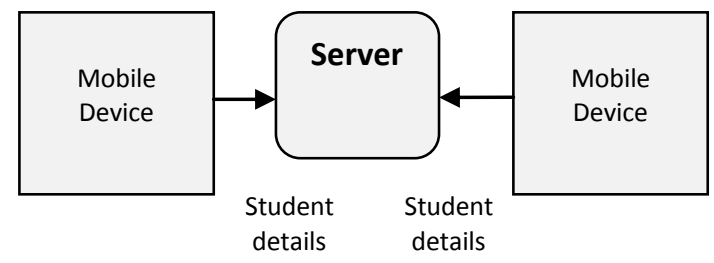

Figure 2: Student Registration

- In the next level, course material is opened by the students, downloadable and viewable in document formats. Otherwise, students can move ahead and take core tests, and subsequent improvement tests.

- If the student chooses to take tests, the server selects and allocates the questions from each chapter. Those questions have to be attempted by the student.

\subsection{Online Test Round}

- The online test portal opens, the test timer starts and students are made to answer multiple choice questions.

- The test can be conducted in a wireless LAN network or a Wi-Fi enabled location e.g., computer labs, test centers.

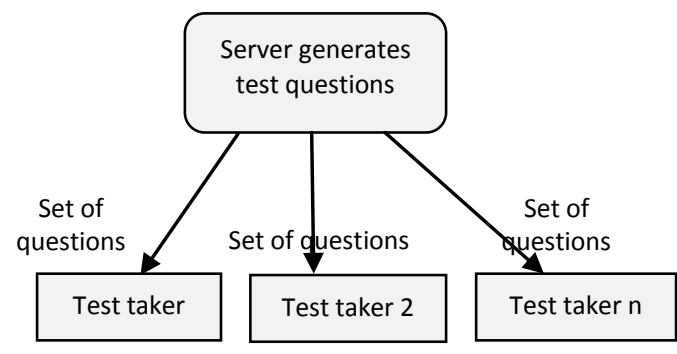

Figure 3: A Wireless Network
- The administrator at the server side selects and assigns the test sections before the test. Students will be made to take the test on those sections only.

- During the test, for each correct answer, the student is awarded 1 mark. For each wrong answer or a blank answer, he/she is given 0 marks.

\subsection{Evaluation Report Generation}

- Once the test is completed, the performance details are sent to the server, which calculates the marks and displays the time taken for each question attempted.

- Core tests are arranged based on difficulty levels. Section wise and chapter wise performances are specified in the individual results page.

- Similarly, category analysis is given, and if the student scores below the cut off mark, then he/she refers to the course content of the subject he/she is weak in.

- After thoroughly studying the concepts, he/she takes the improvement test on that particular chapter. On taking the improvement test, the student is allowed to take the next level of core test, assuming that the student has got enough practice from the improvement test.

- Finally, the student's performance report is sent back to him/her, identifying the areas he/she is already strong in and the areas that need to be strengthened.

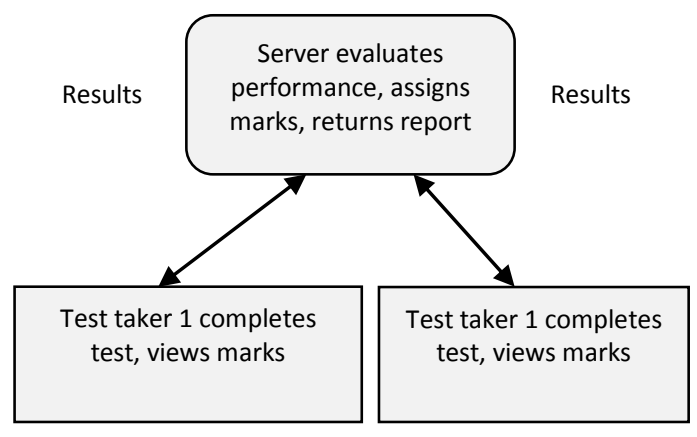

Figure 4: Student's Report with Performance details

\subsection{Group Analysis}

- The results of students, taken collectively, are assessed and then their performances are simulated graphically.

- Intra department and inter departmental analyses are done. Bar graphs and average graphs are used to show department wise and student wise performances. 
Set of questions

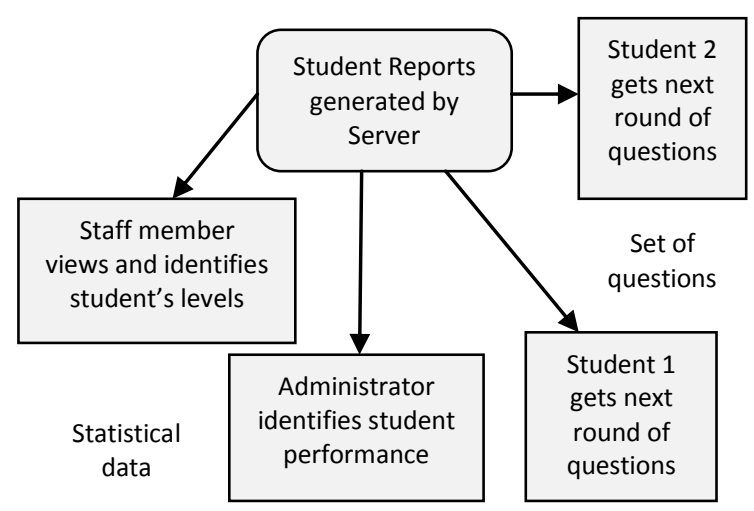

Figure 5: Analysis for Record Keeping

\section{SYSTEM MANAGEMENT}

Our system uses a group of client mobile systems supporting the Android platform. The client module is connected to the server system, in which the database, configured with Microsoft SQL server engine, resides. The server system runs on a Windows Operating System and interacts with the client group through the ASP.NET web application framework. The portal operates in a Wi-Fi environment, and each student maintains an authorized account in it. Figure 6 shows the functions of the client and the server components.

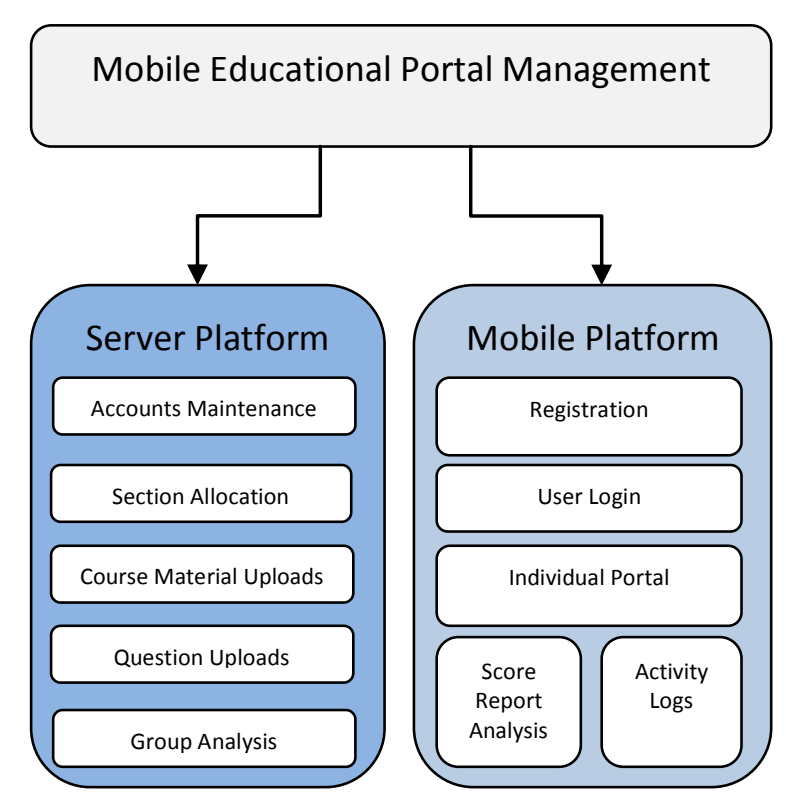

Figure 6: Portal Management System

\subsection{Connecting the Client with the Server}

The database is stored along with the server system, in a remote environment, and the client may be connected to it through a Wi-Fi network. This interconnection would require permission from the Internet for the communication process. If the same is granted, the connections would remain intact throughout the entire process flow, else the request would be bounced back, wherein it is usually followed by subsequent reconnection attempts. This careful authentication on the side of the Internet network addresses a privacy issue.

The core component of the Android system is a manifest XML (Extensible Markup Language) file, which maintains essential information about the application. This file contains the XML command for the Internet permission request, the "android.permission.ACCESS_NETWORK_STATE", and this command helps to access the parent class called ConnectivityManager. This class monitors network connections such as Wi-Fi and GPRS, and answers queries related to them.

In order to connect with the database, it is needed to procure the JDBC (Java Database Connectivity) driver library for SQL server, called the jTDS driver. It is a purely Java based driver, compatible with Android, and it is usually specified in the connection URL, to post into and retrieve entries from the SQL database. Interestingly, SQL server sustains a very granular user level security feature, and this makes it very suitable for business and critical data applications. Usually the data items in the client side are pushed out of the Android platform, converted into XML format, and then passed on to the SQL server engine, through the connection string. Similarly in the server side, ASP.NET controls the data transmission to and from the SQL server engine.

Course materials are uploaded by the administrator into the database from the server system. When a client tries to access this online content through the device, FTP (File Transfer Protocol) presides over the transfer of files and documents between the device and the server.

Thus in the entire system, the client and the server are connected with each other, through the intermediate SQL server database. This course management system could also be hosted in a similar manner, this time, using Web Services as a bridge between the client and the server.

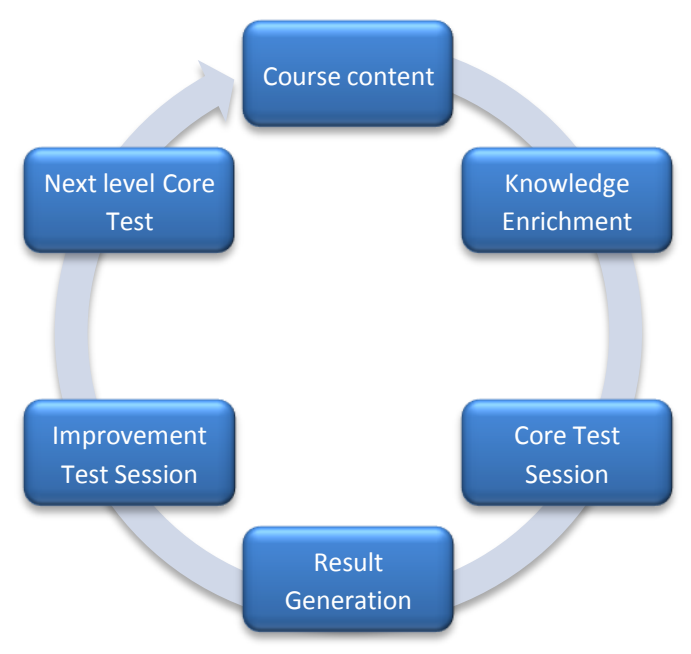

Figure 7: Iterative Learning Process

Figure 7 shows the repetitive series of tests and study sessions which a student undergoes, and the motive behind this process 
it to enhance his/her proficiency in a particular subject he/she studies, and to master his/her skills in it.

\section{RESULTS AND ANALYSIS}

The student accesses course content related to his/her study, viewable in the device and then advances to take the test. The departments of study under consideration are CSE (Computer Science and Engineering), ECE (Electronics and Communication Engineering), EEE (Electrical and Electronics Engineering) and ME (Mechanical Engineering). When the test is completed, the student's particulars, the question parameters such as the chapter name, correct answer, the chosen answer and the time taken, are displayed. A score summary containing the section wise (i.e., quantitative, verbal and technical section) marks, and the category analysis showing the marks allocated and the marks scored for each chapter, under each section, are presented. The score report for the improvement test under each chapter is generated in a similar manner. Table 1 shows a sample result page.

Table 1.Result Generation Page

\begin{tabular}{|c|c|c|c|c|}
\hline & \multicolumn{4}{|c|}{$\begin{array}{ll}\text { Test Name } & : \text { Core Test } 1 \\
\text { Student Name } & \text { John } \\
\text { Exam Date } & : 01 / 08 / 2013 \\
\text { Department } & : \text { CSE } \\
\end{array}$} \\
\hline \multicolumn{5}{|c|}{ Score Summary } \\
\hline Section 1 & Quantitative & \multicolumn{2}{|c|}{$\begin{array}{l}\text { Marks } \\
\text { Scored }\end{array}$} & 10 \\
\hline Section 2 & Verbal & \multicolumn{2}{|c|}{$\begin{array}{l}\text { Marks } \\
\text { Scored }\end{array}$} & 2 \\
\hline Section 3 & Technical & \multicolumn{2}{|c|}{$\begin{array}{l}\text { Marks } \\
\text { Scored }\end{array}$} & 9 \\
\hline \multicolumn{5}{|c|}{ Total Marks } \\
\hline Section 1 & \multicolumn{4}{|c|}{ Quantitative } \\
\hline $\begin{array}{l}\text { Question } \\
\text { Number }\end{array}$ & $\begin{array}{l}\text { Chapter } \\
\text { Name }\end{array}$ & $\begin{array}{l}\text { Correct } \\
\text { Answer }\end{array}$ & $\begin{array}{c}\text { Your } \\
\text { Answer }\end{array}$ & $\begin{array}{c}\text { Time } \\
\text { Taken } \\
\text { (s) }\end{array}$ \\
\hline 1 & $\begin{array}{l}\text { Time and } \\
\text { Work }\end{array}$ & A & A & 56 \\
\hline 2 & $\begin{array}{l}\text { Number } \\
\text { Theory }\end{array}$ & $\mathrm{D}$ & $\mathrm{D}$ & 50 \\
\hline 3 & $\begin{array}{l}\text { Number } \\
\text { Theory }\end{array}$ & B & B & 60 \\
\hline 4 & $\begin{array}{l}\text { Time and } \\
\text { Work }\end{array}$ & $\mathrm{C}$ & $\mathrm{C}$ & 65 \\
\hline 5 & $\begin{array}{l}\text { Time and } \\
\text { Work }\end{array}$ & $\mathrm{D}$ & $\mathrm{D}$ & 70 \\
\hline 6 & $\begin{array}{l}\text { Number } \\
\text { Theory }\end{array}$ & B & B & 150 \\
\hline 7 & $\begin{array}{l}\text { Time and } \\
\text { Work }\end{array}$ & B & B & 240 \\
\hline 8 & $\begin{array}{l}\text { Number } \\
\text { Theory }\end{array}$ & $\mathrm{C}$ & $\mathrm{C}$ & 80 \\
\hline 9 & $\begin{array}{c}\text { Number } \\
\text { Theory }\end{array}$ & $\mathrm{D}$ & $\mathrm{D}$ & 100 \\
\hline 10 & $\begin{array}{l}\text { Time and } \\
\text { Work }\end{array}$ & A & A & 95 \\
\hline Section 1 & \multicolumn{4}{|c|}{ Category Analysis } \\
\hline
\end{tabular}

\begin{tabular}{|c|c|c|c|c|}
\hline $\begin{array}{l}\text { Chapter } \\
\text { Name }\end{array}$ & $\begin{array}{l}\text { Marks } \\
\text { Scored }\end{array}$ & \multicolumn{3}{|c|}{ Maximum Marks } \\
\hline $\begin{array}{l}\text { Time and } \\
\text { Work }\end{array}$ & 10 & \multicolumn{3}{|c|}{10} \\
\hline $\begin{array}{c}\text { Number } \\
\text { Theory }\end{array}$ & 10 & \multicolumn{3}{|c|}{10} \\
\hline Section 2 & \multicolumn{4}{|c|}{ Verbal } \\
\hline $\begin{array}{l}\text { Question } \\
\text { Number }\end{array}$ & $\begin{array}{c}\text { Chapter } \\
\text { Name }\end{array}$ & $\begin{array}{l}\text { Correct } \\
\text { Answer }\end{array}$ & $\begin{array}{c}\text { Your } \\
\text { Answer }\end{array}$ & $\begin{array}{c}\text { Time } \\
\text { Taken } \\
\text { (s) }\end{array}$ \\
\hline 1 & $\begin{array}{c}\text { Critical } \\
\text { Reasoning }\end{array}$ & B & A & 65 \\
\hline 2 & $\begin{array}{c}\text { Verbal } \\
\text { Analogies }\end{array}$ & $\mathrm{D}$ & $\mathrm{C}$ & 54 \\
\hline 3 & $\begin{array}{c}\text { Critical } \\
\text { Reasoning }\end{array}$ & $\mathrm{C}$ & $\mathrm{C}$ & 42 \\
\hline 4 & $\begin{array}{c}\text { Critical } \\
\text { Reasoning }\end{array}$ & $\mathrm{C}$ & $\mathrm{D}$ & 120 \\
\hline 5 & $\begin{array}{c}\text { Verbal } \\
\text { Analogies }\end{array}$ & $\mathrm{D}$ & $\mathrm{D}$ & 125 \\
\hline 6 & $\begin{array}{c}\text { Verbal } \\
\text { Analogies }\end{array}$ & A & B & 107 \\
\hline 7 & $\begin{array}{c}\text { Critical } \\
\text { Reasoning }\end{array}$ & B & $\mathrm{C}$ & 110 \\
\hline 8 & $\begin{array}{c}\text { Verbal } \\
\text { Analogies }\end{array}$ & A & A & 87 \\
\hline 9 & $\begin{array}{c}\text { Verbal } \\
\text { Analogies }\end{array}$ & B & $\mathrm{D}$ & 93 \\
\hline 10 & $\begin{array}{c}\text { Critical } \\
\text { Reasoning }\end{array}$ & $\mathrm{C}$ & B & 101 \\
\hline Section 2 & \multicolumn{4}{|c|}{ Category Analysis } \\
\hline $\begin{array}{l}\text { Chapter } \\
\text { Name }\end{array}$ & $\begin{array}{l}\text { Marks } \\
\text { Scored }\end{array}$ & \multicolumn{3}{|c|}{ Maximum Marks } \\
\hline $\begin{array}{c}\text { Critical } \\
\text { Reasoning }\end{array}$ & 1 & \multicolumn{3}{|c|}{10} \\
\hline $\begin{array}{c}\text { Verbal } \\
\text { Analogies }\end{array}$ & 2 & \multicolumn{3}{|c|}{10} \\
\hline Section 3 & \multicolumn{4}{|c|}{ Technical } \\
\hline $\begin{array}{l}\text { Question } \\
\text { Number }\end{array}$ & $\begin{array}{l}\text { Chapter } \\
\text { Name }\end{array}$ & $\begin{array}{l}\text { Correct } \\
\text { Answer }\end{array}$ & $\begin{array}{c}\text { Your } \\
\text { Answer }\end{array}$ & $\begin{array}{c}\text { Time } \\
\text { Taken } \\
\text { (s) }\end{array}$ \\
\hline 1 & $\begin{array}{l}\text { Network } \\
\text { Protocols }\end{array}$ & $\mathrm{D}$ & $\mathrm{D}$ & 57 \\
\hline 2 & $\begin{array}{l}\text { Network } \\
\text { Protocols }\end{array}$ & $\mathrm{D}$ & A & 69 \\
\hline 3 & $\begin{array}{c}\text { Data } \\
\text { Structures }\end{array}$ & $\mathrm{C}$ & $\mathrm{C}$ & 84 \\
\hline 4 & $\begin{array}{l}\text { Network } \\
\text { Protocols }\end{array}$ & B & B & 87 \\
\hline 5 & $\begin{array}{c}\text { Data } \\
\text { Structures }\end{array}$ & A & A & 99 \\
\hline 6 & $\begin{array}{c}\text { Data } \\
\text { Structures }\end{array}$ & $\mathrm{C}$ & B & 107 \\
\hline 7 & $\begin{array}{l}\text { Network } \\
\text { Protocols }\end{array}$ & $\mathrm{D}$ & $\mathrm{D}$ & 123 \\
\hline 8 & $\begin{array}{c}\text { Data } \\
\text { Structures }\end{array}$ & $\mathrm{C}$ & $\mathrm{D}$ & 43 \\
\hline 9 & $\begin{array}{c}\text { Data } \\
\text { Structures }\end{array}$ & A & A & 88 \\
\hline 10 & $\begin{array}{l}\text { Network } \\
\text { Protocols }\end{array}$ & $\mathrm{C}$ & $\mathrm{C}$ & 156 \\
\hline
\end{tabular}




\begin{tabular}{|c|c|c|}
\hline Section 3 & \multicolumn{2}{|c|}{ Category Analysis } \\
\hline $\begin{array}{c}\text { Chapter } \\
\text { Name }\end{array}$ & $\begin{array}{c}\text { Marks } \\
\text { Scored }\end{array}$ & Maximum Marks \\
\hline $\begin{array}{c}\text { Data } \\
\text { Structures }\end{array}$ & 3 & 10 \\
\hline
\end{tabular}

\begin{tabular}{|c|c|c|}
\hline $\begin{array}{c}\text { Network } \\
\text { Protocols }\end{array}$ & 4 & 10 \\
\hline
\end{tabular}

Table 2.Activity Log

\begin{tabular}{|c|c|c|c|c|c|c|c|c|}
\hline Date & Test Name & $\begin{array}{c}\text { Section 1 } \\
\text { Name }\end{array}$ & $\begin{array}{c}\text { Section 1 } \\
\text { Mark }\end{array}$ & $\begin{array}{c}\text { Section 2 } \\
\text { Name }\end{array}$ & $\begin{array}{c}\text { Section 2 } \\
\text { Mark }\end{array}$ & $\begin{array}{c}\text { Section 3 } \\
\text { Name }\end{array}$ & $\begin{array}{c}\text { Section 3 } \\
\text { Mark }\end{array}$ & $\begin{array}{c}\text { Total } \\
\text { Mark }\end{array}$ \\
\hline $01 / 08 / 2013$ & Core Test 1 & Quantitative & 10 & Verbal & 3 & Technical & 7 & 20 \\
\hline $04 / 08 / 2013$ & Core Test 2 & Technical & 9 & Quantitative & 8 & Verbal & 8 & 25 \\
\hline $08 / 08 / 2013$ & Core Test 3 & Verbal & 9 & Technical & 10 & Quantitative & 10 & 29 \\
\hline
\end{tabular}

Table 2 is an instance of the Activity Log for the student, which he/she could use to view his/her progress from time to time. It maintains a record of the tests taken, the section wise marks and the total marks scored.

The Graphical view displays the marks scored in each section for a core test, graphically, in a student's device, as shown in Figure 8 . The $x$-axis represents the individual tests, and the marks secured in the corresponding test are taken in the yaxis.

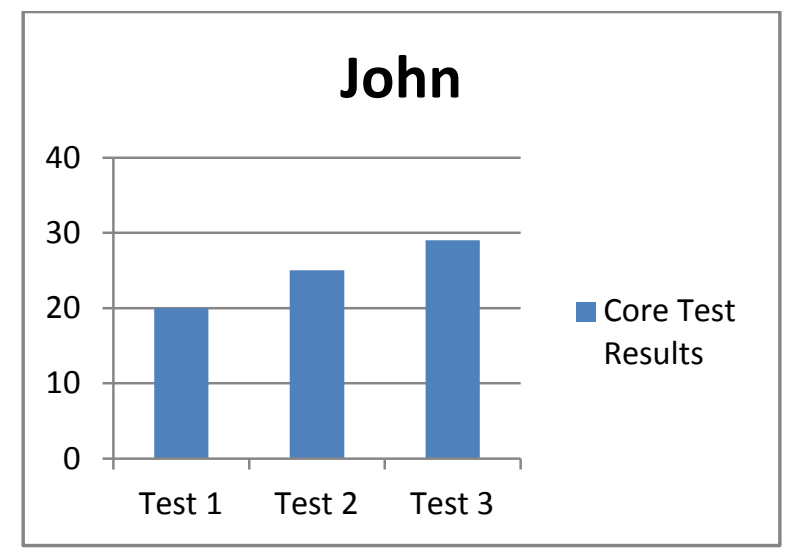

Figure 8: Individual Graphical View

Now, the server would be able to view the performances in the tests, within the department with the help of the registration numbers, unique to each student. Using the graph shown in Figure 9, the examination controller, or even professors and other staff members can analyze each student's performance and level of understanding.

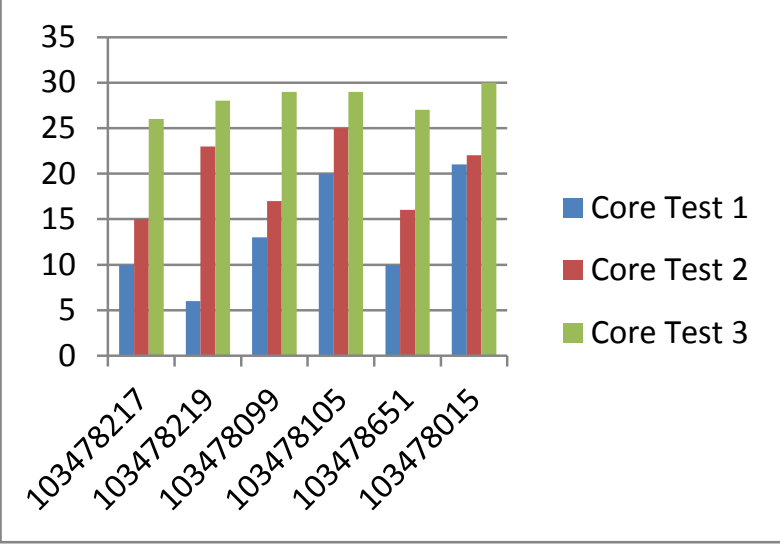

Figure 9: Intra Departmental Analyses for Core Test 1, Core Test 2 and Core Test 3-CSE Department

In the graph, the $\mathrm{x}$-axis represents the registration numbers (For example, 10348219, 103478105 etc.), representing each student. The y-axis represents the marks scored.

This analysis helps them to take necessary steps to further motivate or improvise a student. If the student's mark is lower than the assigned cut off, he/she can then refer to and study the course material related to the chapter he/she is weak in, and can subsequently take improvement tests.

Next, the server can view the comparative results among the departments as a whole. The departments are taken in the $\mathrm{x}$ axis, and the total marks secured are taken in the y-axis. The test marks obtained for all the three tests are shown graphically in Figure 10. 


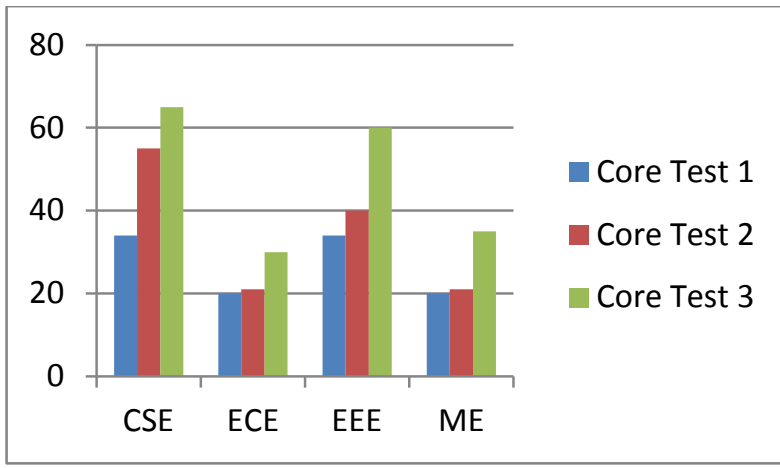

Figure 10: Intra Departmental Analyses for Core Test 1, Core Test 2 and Core Test 3

The average score for all the 3 tests, for each department is generated in a tabular format, as shown in Table 3.

Table 3.Group Analysis

\begin{tabular}{|c|c|}
\hline Department & Average Score \\
\hline CSE & 51.3 \\
\hline ECE & 23.6 \\
\hline EEE & 44.6 \\
\hline ME & 25.3 \\
\hline
\end{tabular}

Based on these group results, the academicians and professors can make precise conclusions about the level of difficulty of solving the questions allocated, the department students who performed best, the department students who need improvement and so on.

\section{CONCLUSION}

The online educational portal system is thus established to facilitate students to access online course content, take tests and analyze their performances. The role played by the mobile platform is to enable students take tests in their mobile devices in a wireless compatible form, using the required connectivity protocols. The result page generated for each student shows the performance in each question, the correct answer for that question, the time spent for it and the marks secured, in comparison to the cut off marks. Using this, students get a thorough idea about what course content they need to refer to, the chapters they need to focus on, and how can they master concepts they are weak in.

The paper portrays a client-server architecture in which the business logic analyzes and delivers necessary support to students to improve marks. This also extends an opportunity for students, to strengthen their knowledge over a particular study subject. This concept can be actualized in educational institutions to keep track of their students' capabilities.

\section{FUTURE ENHANCEMENT}

This concept could be implemented in a cloud based web hosting service, for easy access to educational institutions. Depending upon the performance, the scalability (i.e., number of clients) can be extended, so as to encourage more students to make use of this online educational portal. As a result, one could incorporate this in real time critical educational applications where students across various geographical borders can participate and enlighten themselves.

In addition, one can determine a student's performance by using the time taken by him/her to attempt an individual question, an entire chapter, an entire section, and in turn, an entire test. Based on this duration, the student's response, the comparison between the duration taken by him/her and by another student, one can infer whether he/she is strong or he/she needs to strengthen himself/herself in the chapter in question. This calculation may be done using mobile analytics. Besides, based on the marks scored by individual students, they can be categorized into groups, i.e., students scoring within a particular range of marks can be grouped under one category. Similarly, students scoring within a different range of marks can be grouped accordingly.

Finally, one can apply this concept as a mobile MOOC (Massive Open Online Course), so that participants can engage in a networked learning, using wireless devices. Offering course materials and building up networks such as user forums and peer review communities in a mobile mode ensures a more proactive participation, with quicker and effective results.

\section{ACKNOWLEDGMENTS}

We express our thanks to our guide, Mr. N.S.N Cailassame. His advices and suggestions during the course of the project development and research activities helped us in preparing this paper.

\section{REFERENCES}

[1] Gupta, S.K.S and Srimani, P.K.(2000), "Experience in teaching a graduate course in mobile computing", In Frontiers in Education Conference, 2000. FIE 2000. 30th Annual, Vol.2.

[2] Ayob, N, Hussin, A.R.C and Dahlan, H.M. (2009), "Three Layers Design Guideline for Mobile Application", In Information Management and Engineering, 2009. ICIME '09, International Conference $(K L)$.

[3] Yi-Bing Lin, Chien-Chun Huang-Fu and Nabil Alrajeh, "Predicting human movement based on telecom's handoff in Mobile networks", In IEEE Transactions on Mobile Computing.

[4] Keqin Li (2011) "Analysis of Distance-Based Location Management in Wireless Communication Networks", In IEEE Transactions on Parallel and Distributed Systems.

[5] C.H. Yun and M.S. Chen (2007), "Mining Mobile Sequential Patterns in a Mobile Commerce Environment, In IEEE Transactions on Systems, Man, and Cybernetics, Part C, vol. 37, no. 2, pp. 278-295.

[6] Eric Hsueh-Chan Lu, Wang-Chien Lee and Vincent S. Tseng(2012), "A Framework for Mobile Commerce Pattern Mining and Prediction", In IEEE Transactions on Knowledge and Data Engineering, Vol. 24, No. 5.

[7] Eric Hsueh-Chan Lu, Vincent S. Tseng, and Philip S. Yu (2011) "Mining Cluster-Based Temporal Mobile Sequential Patterns in Location-Based Service Environments", In IEEE Transactions on Knowledge and Data Engineering, Vol. 23, No. 6.

[8] Stephen Shaoyi Liao, Huai Qing Wang, Qiu Dan Li, and Wei Yi Liu(2006), “A Functional-Dependencies-Based 
Bayesian Networks Learning Method and Its Application in a Mobile Commerce System", In IEEE Transactions on Systems, Man and Cybernetics-Part B: Cybernetics, Vol:36, No.3.

[9] T. Sathyan and M. Hedley, "Fast and Accurate Cooperative Tracking in Wireless Networks", In IEEE Transactions on Mobile Computing.

[10] Hsun-Ping Hsieh, Cheng-Te Li, Shou-De Lin (2011) "BeTracker: A System for Finding Behavioural Patterns from Contextual Sensor and Social Data", In 11th IEEE International Conference on Data Mining Workshops.

[11] Jun-Ki Min and Sung-Bae Cho (2011), "Mobile Human Network Management and Recommendation by Probabilistic Social Mining", In IEEE Transactions on Systems, Man, and Cybernetics-Part B: Cybernetics, Vol. 41, No. 3 .

[12] Jing Yuan, Yu Zheng, Xing Xie, Guangzhong Sun, "TDrive: Enhancing Driving Directions with Taxi Drivers' Intelligence", In IEEE Transactions on Knowledge and Data Engineering.

[13] Mark N. Gasson, Eleni Kosta, Denis Royer, Martin Meints, and Kevin Warwick (2011) "Normality Mining: Privacy Implications of Behavioural Profiles Drawn From GPS Enabled Mobile Phones", In IEEE Transactions on Systems, Man, and Cybernetics-Part C: Applications and Reviews, Vol. 41, No. 2.

[14] Yungeun Kim, Yohan Chon, and Hojung Cha (2012) "Smartphone-Based Collaborative and Autonomous
Radio Fingerprinting", In IEEE Transactions on Systems, Man, and Cybernetics-Part C: Applications and Reviews, Vol. 42, No. 1 .

[15] Kenneth Wai-Ting Leung, Dik Lun Lee, Wang-Chien Lee (2007) "PMSE: A Personalized Mobile Search Engine", In IEEE Transactions in Knowledge and Data Engineering. Latex Class Files, Vol. 6, No. 1.

[16] Li-Der Chou, Nien-Hwa Lai, Yen-Wen Chen, Yao-Jen Chang, Jyun-Yan Yang, Lien-Fu Huang, Wen-Ling Chiang, Hung-Yi Chiu, and Haw-Yun Shin(2011) "Mobile Social Network Services for Families with Children with Developmental Disabilities", In IEEE Transactions on Information Technology in Biomedicine, Vol. 15, No. 4.

[17] Tzung-Shi Chen, Yen-Ssu Chou, and Tzung-Cheng Chen (2012) "Mining User Movement Behaviour Patterns in a Mobile Service Environment", In IEEE Transactions on Systems, Man, and Cybernetics-Part A: Systems and Humans, Vol. 42, No. 1.

[18] Adrian Holzer, Patrick Eugster, and Benoît Garbinato, "Evaluating Implementation Strategies for Locationbased Multicast Addressing", In IEEE Transactions on Mobile Computing.

[19] Liang He, Jianping Pan and Jingdong Xu, "A Progressive Approach to Reducing Data Collection Latency in Wireless Sensor Networks with Mobile Elements", In IEEE Transactions on Mobile Computing. 